\title{
CAPUTO FRACTIONAL DIFFERENTIAL INCLUSIONS OF ARBITRARY ORDER WITH NONLOCAL INTEGRO-MULTIPOINT BOUNDARY CONDITIONS
}

\author{
BASHIR AHMAD, DOA'A GAROUT, SOTIRIS K. NTOUYAS, AND AHMED ALSAEDI
}

Received 18 February, 2017

\begin{abstract}
We study a new class of boundary value problems of Caputo type fractional differential inclusions supplemented with nonlocal integro-multipoint boundary conditions. An existence result for the problem with convex valued (multivalued) map is obtained via nonlinear alternative of Leray-Schauder type, while the existence of solutions for the problem involving nonconvex valued map is established by means of Wegrzyk's fixed point theorem. Our results are well illustrated with examples.
\end{abstract}

2010 Mathematics Subject Classification: 34A60; 34A08; 34A12; 34B15

Keywords: Caputo fractional derivative, fractional differential inclusions, existence, fixed point theorems

\section{INTRODUCTION}

In this paper, we obtain sufficient criteria for the existence of solutions for a Caputo type fractional differential inclusion

$$
{ }^{c} D^{q} x(t) \in F(t, x(t)), n-1<q \leq n, t \in[0,1],
$$

equipped with the boundary conditions

$$
\left\{\begin{array}{l}
x(0)=0, x^{\prime}(0)=0, x^{\prime \prime}(0)=0, \ldots, x^{(n-2)}(0)=0, \\
x(1)=a \int_{0}^{\xi} x(s) d s+b \sum_{i=1}^{m-2} \alpha_{i} x\left(\eta_{i}\right), 0<\xi<\eta_{1}<\eta_{2}<\ldots<\eta_{m-2}<1,
\end{array}\right.
$$

where ${ }^{c} D^{q}$ denotes the Caputo fractional derivative of order $q, F:[0,1] \times \mathbb{R} \rightarrow \mathcal{P}(\mathbb{R})$ is a multi-valued map, $\mathcal{P}(\mathbb{R})$ is a family of all nonempty subsets of $\mathbb{R}, a$ and $b$ are real constants and $\alpha_{i}, i=1, \ldots, m-2$, are positive real constants.

Here we remark that the last condition in (1.2) connecting the nonlocal multi-point and strip conditions can be interpreted as the linear combination of the values of the unknown function at nonlocal points $\eta_{i} \in(0,1)$ together with the strip contribution of the unknown function on an arbitrary segment $(0, \xi) \subset[0,1]$ is proportional to 
the value of the unknown function at $t=1$. The multi-point-strip boundary data, occurring in certain problems of thermodynamics, elasticity and wave propagation $[3,8,27]$, correspond to the situation when the controllers at the end points of the interval dissipate or add energy according to censors located at interior positions (finite many points and strip) of the domain.

In recent years, the topic of initial and boundary value problems involving fractional differential equations and inclusions has received great attention and many authors have contributed to its advancement by producing a variety of results $[2,4-7,9$, $15,18,23,25,28,34]$. The interest in this topic owes to its extensive applications in the mathematical modeling of scientific and applied problems occurring in various disciplines such as ecology, acoustics, viscoelasticity, electromagnetics, control theory and material sciences. An important characteristic, distinguishing fractional order operators from their classical counterparts, is their nonlocal nature that can take care of the past history of the processes and phenomena involved in the problem. For some specific examples, we refer the reader to the works $[20,35]$ and the references cited therein. In [29], the authors studied the existence of solutions for one-dimensional higher-order semi-linear Caputo type fractional differential equations supplemented with nonlocal multi-point discrete and integral boundary conditions.

Differential inclusions (generalization of differential equations and inequalities) are found to be of great utility in studying dynamical systems and stochastic processes. An important application of differential inclusions can be found in the area of sweeping processes. In fact, evolution differential inclusions appear in the mathematical modelling of sweeping processes. For a detailed account of this subject, we refer the reader to the monograph [26] and research articles [1,16]. In [31], it has been shown that the existence and uniqueness of BV continuous sweeping processes can be easily reduced to the Lipschitz continuous case by means of a suitable reparametrization of the associated moving convex set. Differential inclusions also play a key role in the study of granular systems [30,32], nonlinear dynamics of wheeled vehicles [10], control problems [21], etc. In [24], one can find a detailed description of pressing issues in stochastic processes, control, differential games, optimization and their application in finance, manufacturing, queueing networks, and climate control. For application of fractional differential inclusions in synchronization processes, we refer the reader to the paper [13].

The aim of the present work is to develop the existence theory for a multivalued analog of the problem addressed in [29]. The first result dealing with the convex valued maps involved in the given problem is obtained via nonlinear alternative of Leray-Schauder type, while the existence of solutions for the nonconvex valued maps is shown by applying Wegrzyk's fixed point theorem. The main results are presented in Section 3. The preliminary material needed to execute the main work is outlined in Section 2. 


\section{PRELIMINARIES}

First of all, we recall some definitions of fractional calculus [20,35].

Definition 1. The Riemann-Liouville fractional integral of order $\alpha>0$ of a function $g:(0, \infty) \rightarrow \mathbb{R}$ is defined by

$$
J^{\alpha} g(t)=\int_{0}^{t} \frac{(t-s)^{\alpha-1}}{\Gamma(\alpha)} g(s) d s,
$$

provided the right-hand side is point-wise defined on $(0, \infty)$, where $\Gamma$ is the Gamma function.

Definition 2. The Riemann-Liouville fractional derivative of order $\alpha>0$ of a continuous function $g:(0, \infty) \rightarrow \mathbb{R}$ is defined by

$$
D^{\alpha} g(t)=\frac{1}{\Gamma(n-\alpha)}\left(\frac{d}{d t}\right)^{n} \int_{0}^{t} \frac{g(s)}{(t-s)^{\alpha-n+1}} d s, \quad n-1<\alpha<n,
$$

where $n=[\alpha]+1,[\alpha]$ denotes the integer part of real number $\alpha$, provided the righthand side is point-wise defined on $(0, \infty)$.

Definition 3. The Caputo derivative of order $q$ for a function $f:[0, \infty) \rightarrow \mathbb{R}$ can be written as

$$
{ }^{c} D^{q} f(t)=D^{q}\left(f(t)-\sum_{k=0}^{n-1} \frac{t^{k}}{k !} f^{(k)}(0)\right), \quad t>0, \quad n-1<q<n .
$$

Remark 1. If $f(t) \in C^{n}[0, \infty)$, then

$$
{ }^{c} D^{q} f(t)=\frac{1}{\Gamma(n-q)} \int_{0}^{t} \frac{f^{(n)}(s)}{(t-s)^{q+1-n}} d s=I^{n-q} f^{(n)}(t), t>0, n-1<q<n .
$$

In order to define a solution of the given problem, we need the following known result [29].

Lemma 1. Let $\omega \in C([0,1], \mathbb{R})$ and

$$
\Delta=1-\frac{a \xi^{n}}{n}-b \sum_{i=1}^{m-2} \alpha_{i} \eta_{i}^{n-1} \neq 0 .
$$

The boundary value problem

$$
\left\{\begin{array}{l}
{ }^{c} D^{q} x(t)=\omega(t), \quad t \in[0,1], \\
x(0)=0, x^{\prime}(0)=0, x^{\prime \prime}(0)=0, \ldots, x^{(n-2)}(0)=0, \\
x(1)=a \int_{0}^{\xi} x(s) d s+b \sum_{i=1}^{m-2} \alpha_{i} x\left(\eta_{i}\right), 0<\xi<\eta_{1}<\eta_{2}<\ldots<\eta_{m-2}<1,
\end{array}\right.
$$


is equivalent to the following integral equation

$$
\begin{aligned}
x(t)= & \int_{0}^{t} \frac{(t-s)^{q-1}}{\Gamma(q)} \omega(s) d s+\frac{t^{n-1}}{\Delta}\left(a \int_{0}^{\xi} \frac{(\xi-s)^{q}}{\Gamma(q+1)} \omega(s) d s\right. \\
& \left.+b \sum_{i=1}^{m-2} \alpha_{i} \int_{0}^{\eta_{i}} \frac{\left(\eta_{i}-s\right)^{q-1}}{\Gamma(q)} \omega(s) d s-\int_{0}^{1} \frac{(1-s)^{q-1}}{\Gamma(q)} \omega(s) d s\right) .
\end{aligned}
$$

Next we fix our terminology and outline some basic concepts of multivalued analysis $[14,19]$.

Denote by $C([0,1], \mathbb{R})$ the Banach space of all continuous functions from $[0,1]$ into $\mathbb{R}$ endowed with the norm $\|x\|=\sup \{|x(t)|, t \in[0,1]\}$. By $L^{1}([0,1], \mathbb{R})$ we denote the space of Lebesgue measurable and integrable functions $x:[0,1] \rightarrow \mathbb{R}$ such that $\|x\|_{L^{1}}=\int_{0}^{1}|x(t)| d t$.

For a normed space $(X,\|\cdot\|)$, let $\mathcal{P}_{c l}(X)=\{Y \in \mathcal{P}(X): Y$ is closed $\}, \mathscr{P}_{b}(X)=$ $\{Y \in \mathcal{P}(X): Y$ is bounded $\}, \mathcal{P}_{c l, b}(X)=\{Y \in \mathcal{P}(X): Y$ is closed and bounded $\}$, $\mathcal{P}_{c p}(X)=\{Y \in \mathcal{P}(X): Y$ is compact $\}$, and $\mathcal{P}_{c p, c}(X)=\{Y \in \mathcal{P}(X): Y$ is compact and convex $\}$.

A multi-valued map $\mathscr{H}: X \rightarrow \mathcal{P}(X)$ :

(i) is convex (closed) valued if $\mathscr{H}(x)$ is convex (closed) for all $x \in X$.

(ii) is bounded on bounded sets if $\mathscr{H}(Y)=\cup_{x \in Y} \mathscr{H}(x)$ is bounded in $X$ for all $Y \in \mathcal{P}_{b}(X)$ (i.e. $\left.\sup _{x \in Y}\{\sup \{|y|: y \in \mathscr{H}(x)\}\}<\infty\right)$.

(iii) is called upper semi-continuous (u.s.c.) on $X$ if for each $x_{0} \in X$, the set $\mathscr{H}\left(x_{0}\right)$ is a nonempty closed subset of $X$, and if for each open set $N$ of $X$ containing $\mathscr{H}\left(x_{0}\right)$, there exists an open neighborhood $\mathcal{N}_{0}$ of $x_{0}$ such that $\mathscr{H}\left(\mathcal{N}_{0}\right) \subseteq N$.

(iv) $G$ is lower semi-continuous (l.s.c.) if the set $\{y \in X: \mathscr{H}(y) \cap Y \neq \varnothing\}$ is open for any open set $Y$ in $X$.

(v) is said to be completely continuous if $\mathscr{H}(\mathbb{B})$ is relatively compact for every $\mathbb{B} \in \mathcal{P}_{b}(X)$; If the multi-valued map $\mathscr{H}$ is completely continuous with nonempty compact values, then $\mathscr{H}$ is u.s.c. if and only if $\mathscr{H}$ has a closed graph, i.e., $x_{n} \rightarrow x_{*}, y_{n} \rightarrow y_{*}, y_{n} \in \mathscr{H}\left(x_{n}\right)$ imply $y_{*} \in \mathscr{H}\left(x_{*}\right)$.

(vi) is said to be measurable if for every $y \in X$, the function

$$
t \longmapsto d(y, \mathscr{H}(t))=\inf \{|y-z|: z \in \mathscr{H}(t)\}
$$

is measurable.

(vii) has a fixed point if there is $x \in X$ such that $x \in \mathscr{H}(x)$. The fixed point set of the multivalued operator $\mathscr{H}$ will be denoted by Fix $\mathscr{H}$.

\section{EXISTENCE RESULTS}

By Lemma 1, we can define a solution of problem (1.1)-(1.2) as follows. 
Definition 4. A function $x \in C^{n}([0,1], \mathbb{R})$ is a solution of problem (1.1)-(1.2) if there exists a function $f \in L^{1}([0,1], \mathbb{R})$ such that $f(t) \in F(t, x(t))$ a.e. on $[0,1]$ and

$$
\begin{aligned}
x(t)= & \int_{0}^{t} \frac{(t-s)^{q-1}}{\Gamma(q)} f(s) d s+\frac{t^{n-1}}{\Delta}\left(a \int_{0}^{\xi} \frac{(\xi-s)^{q}}{\Gamma(q+1)} f(s) d s\right. \\
& \left.+b \sum_{i=1}^{m-2} \alpha_{i} \int_{0}^{\eta_{i}} \frac{\left(\eta_{i}-s\right)^{q-1}}{\Gamma(q)} f(s) d s-\int_{0}^{1} \frac{(1-s)^{q-1}}{\Gamma(q)} f(s) d s\right) .
\end{aligned}
$$

Next, we define an operator $\mathcal{K}: C([0,1], \mathbb{R}) \rightarrow \mathcal{P}(C([0,1], \mathbb{R}))$ by

$$
\begin{aligned}
\mathcal{K}(x)= & \left\{z \in C([0,1], \mathbb{R}): z(t)=\int_{0}^{t} \frac{(t-s)^{q-1}}{\Gamma(q)} f(s) d s\right. \\
& +\frac{t^{n-1}}{\Delta}\left(a \int_{0}^{\xi} \frac{(\xi-s)^{q}}{\Gamma(q+1)} f(s) d s+b \sum_{i=1}^{m-2} \alpha_{i} \int_{0}^{\eta_{i}} \frac{\left(\eta_{i}-s\right)^{q-1}}{\Gamma(q)} f(s) d s\right. \\
& \left.\left.-\int_{0}^{1} \frac{(1-s)^{q-1}}{\Gamma(q)} f(s) d s\right), f \in S_{F, x}\right\} .
\end{aligned}
$$

Definition 5. A multi-valued map $F:[0,1] \times \mathbb{R} \rightarrow \mathcal{P}(\mathbb{R})$ is said to be Carathéodory if

(i) $t \mapsto F(t, x)$ is measurable for each $x \in \mathbb{R}$ and

(ii) $x \mapsto F(t, x)$ is upper semicontinuous for almost all $t \in[0,1]$.

Further, a Caratheodory function $F$ is called $L^{1}$-Carathéodory if

(iii) for each $a>0$, there exists $\varphi_{a} \in L^{1}\left([0,1], \mathbb{R}^{+}\right)$such that

$$
\|F(t, x)\|=\sup \{|v|: v \in F(t, x)\} \leq \varphi_{a}(t)
$$

for all $\|x\| \leq a$ and for a.e $t \in[0,1]$.

For each $y \in C([0,1], \mathbb{R})$, we define the set of selections of $F$ by

$$
S_{F, y}:=\left\{v \in L^{1}([0,1], \mathbb{R}): v(t) \in F(t, y(t)) \text { for a.e. } t \in[0,1]\right\} .
$$

\subsection{The convex-valued (Carathéodory) case}

In this subsection, we prove an existence result for problem (1.1)-(1.2), assuming that $F$ is Carathéodory ( $F$ has convex values).

Before presenting the main result, let us state the auxiliary lemmas.

Lemma 2 (Nonlinear alternative of Leray-Schauder type [17]). Let $C$ be a convex set in a normed space, and $E \subset C$ be open subset with $0 \in E$. Then each upper semicontinuous and compact mapping $\mathscr{E}: \bar{E} \rightarrow P(C)$ with compact convex values that is fixed point free on $\partial E$ has at least one of the following two properties: 
(a) $\mathcal{G}$ has a fixed point in $\bar{E}$, or

(b) there exist $x \in \partial E$ and $\lambda \in(0,1)$ such that $x \in \lambda \mathscr{E}(x)$.

Lemma 3 ([22]). Let $X$ be a separable Banach space. Let $F:[0,1] \times \mathbb{R} \rightarrow$ $\mathcal{P}_{c p, c}(X)$ be an $L^{1}-$ Caratheodory multi-valued map, and let $\phi$ be a linear continuous mapping from $L^{1}([0,1], X)$ to $C([0,1], X)$. Then the operator

$\phi \circ S_{F}: C([0,1], X) \longrightarrow \mathcal{P}_{c p, c}(C([0,1], X)), x \mapsto\left(\phi \circ S_{F}\right)(x)=\phi\left(S_{F, x}\right)$, is a closed graph operator in $C([0,1], X) \times C([0,1], X)$.

Theorem 1. Assume that

$\left(H_{1}\right) F:[0,1] \times \mathbb{R} \rightarrow \mathcal{P}(\mathbb{R})$ is $L^{1}-$ Carathéodory and has compact and convex values;

$\left(H_{2}\right)$ there exist a continuous nondecreasing function $\chi:[0, \infty) \rightarrow(0, \infty)$ and a function $k \in C\left([0,1], \mathbb{R}^{+}\right)$such that

$$
\|F(t, x)\|_{\mathcal{P}}:=\sup \{|y|: y \in F(t, x)\} \leq k(t) \chi(\|x\|) \text { for all }(t, x) \in[0,1] \times \mathbb{R} ;
$$

$\left(H_{3}\right)$ There exists a number $N>0$ such that

$$
\frac{N}{\chi(N)\|k\|}\left\{\frac{1}{\Gamma(q+1)}\left[1+\frac{1}{|\Delta|}\left(1+|a| \frac{\xi^{q+1}}{(q+1)}+|b| \sum_{i=1}^{m-2} \alpha_{i} \eta_{i}^{q}\right)\right]\right\}^{-1}>1,
$$

where $\Delta$ is given by (2.1).

Then the problem (1.1)-(1.2) has at least one solution on $[0,1]$.

Proof. We transform the problem (1.1)-(1.2) into a fixed point theorem by considering the operator $\mathcal{K}$ defined by (3.2). It is obvious that the fixed points of $\mathcal{K}$ are solutions of the boundary value problem (1.1)-(1.2). We will show that $\mathcal{K}$ satisfies the assumptions of Leray-Schauder nonlinear alternative. In the first step, we will show that $\mathcal{K}(x)$ is convex for each $x \in C([0,1], \mathbb{R})$. Let $z_{1}, z_{2} \in \mathcal{K}(x)$, then there exist $f_{1}, f_{2} \in S_{F, x}$ such that for each $t \in[0,1]$, we have

$$
\begin{aligned}
z_{i}(t)= & \int_{0}^{t} \frac{(t-s)^{q-1}}{\Gamma(q)} f_{i}(s) d s+\frac{t^{n-1}}{\Delta}\left(a \int_{0}^{\xi} \frac{(\xi-s)^{q}}{\Gamma(q+1)} f_{i}(s) d s\right. \\
& \left.+b \sum_{i=1}^{m-2} \alpha_{i} \int_{0}^{\eta_{i}} \frac{\left(\eta_{i}-s\right)^{q-1}}{\Gamma(q)} f_{i}(s) d s-\int_{0}^{1} \frac{(1-s)^{q-1}}{\Gamma(q)} f_{i}(s) d s\right), i=1,2 .
\end{aligned}
$$

Set $0 \leq \delta \leq 1$, then for each $t \in[0,1]$, we have

$$
\begin{aligned}
{\left[\delta z_{1}+(1-\delta) z_{2}\right](t)=} & \int_{0}^{t} \frac{(t-s)^{q-1}}{\Gamma(q)}\left[\delta f_{1}(s)+(1-\delta) f_{2}(s)\right] d s \\
& +\frac{t^{n-1}}{\Delta}\left(a \int_{0}^{\xi} \frac{(\xi-s)^{q}}{\Gamma(q+1)}\left[\delta f_{1}(s)+(1-\delta) f_{2}(s)\right] d s\right.
\end{aligned}
$$




$$
\begin{aligned}
& +b \sum_{i=1}^{m-2} \alpha_{i} \int_{0}^{\eta_{i}} \frac{\left(\eta_{i}-s\right)^{q-1}}{\Gamma(q)}\left[\delta f_{1}(s)+(1-\delta) f_{2}(s)\right] d s \\
& \left.-\int_{0}^{1} \frac{(1-s)^{q-1}}{\Gamma(q)}\left[\delta f_{1}(s)+(1-\delta) f_{2}(s)\right] d s\right) .
\end{aligned}
$$

Hence, by the convexity of $S_{F, x}$, it follows that $\delta z_{1}+(1-\delta) z_{2} \in \mathcal{K}(x)$.

Now, we show that $\mathcal{K}$ maps bounded sets into bounded sets in $C([0,1], \mathbb{R})$. For $\rho>0$, let $B_{\rho}=\{x \in C([0,1], \mathbb{R}):\|x\| \leq \rho\}$ be a bounded set in $C([0,1], \mathbb{R})$. Thus, for each $z \in \mathcal{K}(x), x \in B_{\rho}$, there exists $f \in S_{F, x}$ such that

$$
\begin{aligned}
z(t)= & \int_{0}^{t} \frac{(t-s)^{q-1}}{\Gamma(q)} f(s) d s+\frac{t^{n-1}}{\Delta}\left(a \int_{0}^{\xi} \frac{(\xi-s)^{q}}{\Gamma(q+1)} f(s) d s\right. \\
& \left.+b \sum_{i=1}^{m-2} \alpha_{i} \int_{0}^{\eta_{i}} \frac{\left(\eta_{i}-s\right)^{q-1}}{\Gamma(q)} f(s) d s-\int_{0}^{1} \frac{(1-s)^{q-1}}{\Gamma(q)} f(s) d s\right) .
\end{aligned}
$$

Then, for $x \in B_{\rho}$, in view of $\left(H_{2}\right)$, we obtain

$$
\begin{aligned}
& |z(t)| \\
& \leq \int_{0}^{t} \frac{(t-s)^{q-1}}{\Gamma(q)} k(s) \chi(\|x\|) d s+\frac{t^{n-1}}{|\Delta|}\left(|a| \int_{0}^{\xi} \frac{(\xi-s)^{q}}{\Gamma(q+1)} k(s) \chi(\|x\|) d s\right. \\
& \left.\quad+|b| \sum_{i=1}^{m-2} \alpha_{i} \int_{0}^{\eta_{i}} \frac{\left(\eta_{i}-s\right)^{q-1}}{\Gamma(q)} k(s) \chi(\|x\|) d s+\int_{0}^{1} \frac{(1-s)^{q-1}}{\Gamma(q)} k(s) \chi(\|x\|) d s\right),
\end{aligned}
$$

which implies that

$$
\|z\| \leq \frac{\chi(\rho)\|k\|}{\Gamma(q+1)}\left(1+\frac{1}{|\Delta|}\left[1+|a| \frac{\xi^{q+1}}{(q+1)}+|b| \sum_{i=1}^{m-2} \alpha_{i} \eta_{i}^{q}\right]\right) .
$$

Next, we show that $\mathcal{K}$ maps bounded sets into equicontinuous sets in $C([0,1], \mathbb{R})$. Let $t_{1}, t_{2} \in[0,1]$ with $t_{1}<t_{2}$ and $x \in B_{\rho}$, then, we obtain for each $z \in \mathcal{K}(x)$

$$
\begin{aligned}
& \left|z\left(t_{2}\right)-z\left(t_{1}\right)\right| \\
& =\mid \int_{0}^{t_{2}} \frac{\left(t_{2}-s\right)^{q-1}}{\Gamma(q)} f(s) d s-\int_{0}^{t_{1}} \frac{\left(t_{1}-s\right)^{q-1}}{\Gamma(q)} f(s) d s+\frac{\left(t_{2}^{n-1}-t_{1}^{n-1}\right)}{\Delta} \times \\
& \times\left[a \int_{0}^{\xi} \frac{(\xi-s)^{q}}{\Gamma(q+1)} f(s) d s+b \sum_{i=1}^{m-2} \alpha_{i} \int_{0}^{\eta_{i}} \frac{\left(\eta_{i}-s\right)^{q-1}}{\Gamma(q)} f(s) d s\right.
\end{aligned}
$$




$$
\begin{aligned}
& \left.-\int_{0}^{1} \frac{(1-s)^{q-1}}{\Gamma(q)} f(s) d s\right] \mid \\
\leq & \left|\int_{0}^{t_{1}} \frac{\left(t_{2}-s\right)^{q-1}-\left(t_{1}-s\right)^{q-1}}{\Gamma(q)} f(s) d s+\int_{t_{1}}^{t_{2}} \frac{\left(t_{2}-s\right)^{q-1}}{\Gamma(q)} f(s) d s\right| \\
& +\left|\frac{t_{2}^{n-1}-t_{1}^{n-1}}{\Delta}\right|\left[|a| \int_{0}^{\xi} \frac{(\xi-s)^{q}}{\Gamma(q+1)}|f(s)| d s\right. \\
& \left.+|b| \sum_{i=1}^{m-2} \alpha_{i} \int_{0}^{\eta_{i}} \frac{\left(\eta_{i}-s\right)^{q-1}}{\Gamma(q)}|f(s)| d s+\int_{0}^{1} \frac{(1-s)^{q-1}}{\Gamma(q)}|f(s)| d s\right] \\
\leq & \chi(\rho)\|k\|\left[\frac{\left|t_{2}^{q}-t_{1}^{q}\right|+2\left(t_{2}-t_{1}\right)^{q}}{\Gamma(q+1)}\right. \\
& \left.+\frac{\left|t_{2}^{n-1}-t_{1}^{n-1}\right|}{\Gamma(q+1)|\Delta|}\left(1+|a| \frac{\xi^{q+1}}{(q+1)}+|b| \sum_{i=1}^{m-2} \alpha_{i} \eta_{i}^{q}\right)\right],
\end{aligned}
$$

which tends to zero independent of $x \in B_{\rho}$ as $\left(t_{2}-t_{1}\right) \rightarrow 0$. Consequently, by the Arzelá-Ascoli theorem, the operator $\mathcal{K}$ is completely continuous.

Since $\mathcal{K}$ is completely continuous, in order to prove that it is u.s.c. it is enough to prove that it has a closed graph. Thus, now, we want to show that $\mathcal{K}$ has a closed graph. Let $x_{n} \rightarrow \hat{x}, z_{n} \in \mathcal{K}\left(x_{n}\right)$, and $z_{n} \rightarrow \hat{z}$. We have to show that $\hat{z} \in \mathcal{K}(\hat{x})$. So, for $z_{n} \in \mathcal{K}\left(x_{n}\right)$, there exists $f_{n} \in S_{F, x_{n}}$ such that for all $t \in[0,1]$, we have

$$
\begin{aligned}
z_{n}(t)= & \int_{0}^{t} \frac{(t-s)^{q-1}}{\Gamma(q)} f_{n}(s) d s+\frac{t^{n-1}}{\Delta}\left(a \int_{0}^{\xi} \frac{(\xi-s)^{q}}{\Gamma(q+1)} f_{n}(s) d s\right. \\
& \left.+b \sum_{i=1}^{m-2} \alpha_{i} \int_{0}^{\eta_{i}} \frac{\left(\eta_{i}-s\right)^{q-1}}{\Gamma(q)} f_{n}(s) d s-\int_{0}^{1} \frac{(1-s)^{q-1}}{\Gamma(q)} f_{n}(s) d s\right)
\end{aligned}
$$

Thus, we have to show that there exists $\hat{f} \in S_{F, \hat{x}}$ such that for each $t \in[0,1]$,

$$
\begin{aligned}
\hat{z}(t)= & \int_{0}^{t} \frac{(t-s)^{q-1}}{\Gamma(q)} \hat{f}(s) d s+\frac{t^{n-1}}{\Delta}\left(a \int_{0}^{\xi} \frac{(\xi-s)^{q}}{\Gamma(q+1)} \hat{f}(s) d s\right. \\
& \left.+b \sum_{i=1}^{m-2} \alpha_{i} \int_{0}^{\eta_{i}} \frac{\left(\eta_{i}-s\right)^{q-1}}{\Gamma(q)} \hat{f}(s) d s-\int_{0}^{1} \frac{(1-s)^{q-1}}{\Gamma(q)} \hat{f}(s) d s\right)
\end{aligned}
$$


Consider the continuous linear operator $\Phi: L^{1}([0,1], \mathbb{R}) \rightarrow C([0,1], \mathbb{R})$ given by

$$
\begin{aligned}
f \mapsto \Phi(f)(t)= & \int_{0}^{t} \frac{(t-s)^{q-1}}{\Gamma(q)} f(s) d s+\frac{t^{n-1}}{\Delta}\left(a \int_{0}^{\xi} \frac{(\xi-s)^{q}}{\Gamma(q+1)} f(s) d s\right. \\
& \left.+b \sum_{i=1}^{m-2} \alpha_{i} \int_{0}^{\eta_{i}} \frac{\left(\eta_{i}-s\right)^{q-1}}{\Gamma(q)} f(s) d s-\int_{0}^{1} \frac{(1-s)^{q-1}}{\Gamma(q)} f(s) d s\right) .
\end{aligned}
$$

Observe that

$$
\begin{aligned}
& \left\|z_{n}(t)-\hat{z}(t)\right\| \\
& =\| \int_{0}^{t} \frac{(t-s)^{q-1}}{\Gamma(q)}\left(f_{n}(s)-\hat{f}(s)\right) d s+\frac{t^{n-1}}{\Delta}\left(a \int_{0}^{\xi} \frac{(\xi-s)^{q}}{\Gamma(q+1)}\left(f_{n}(s)-\hat{f}(s)\right) d s\right. \\
& \quad+b \sum_{i=1}^{m-2} \alpha_{i} \int_{0}^{\eta_{i}} \frac{\left(\eta_{i}-s\right)^{q-1}}{\Gamma(q)}\left(f_{n}(s)-\hat{f}(s)\right) d s \\
& \left.\quad-\int_{0}^{1} \frac{(1-s)^{q-1}}{\Gamma(q)}\left(f_{n}(s)-\hat{f}(s)\right) d s\right) \|
\end{aligned}
$$

tends to 0 as $n \rightarrow \infty$. Thus, it follows by Lemma 3 that $\Phi \circ S_{F}$ is a closed graph operator. Moreover, we have $z_{n}(t) \in \Phi\left(S_{F, x_{n}}\right)$. Since $x_{n} \rightarrow \hat{x}$, we have then

$$
\begin{aligned}
\hat{z}(t)= & \int_{0}^{t} \frac{(t-s)^{q-1}}{\Gamma(q)} \hat{f}(s) d s+\frac{t^{n-1}}{\Delta}\left(a \int_{0}^{\xi} \frac{(\xi-s)^{q}}{\Gamma(q+1)} \hat{f}(s) d s\right. \\
& \left.+b \sum_{i=1}^{m-2} \alpha_{i} \int_{0}^{\eta_{i}} \frac{\left(\eta_{i}-s\right)^{q-1}}{\Gamma(q)} \hat{f}(s) d s-\int_{0}^{1} \frac{(1-s)^{q-1}}{\Gamma(q)} \hat{f}(s) d s\right),
\end{aligned}
$$

for some $\hat{f} \in S_{F, \hat{x}}$.

Finally, we show there exists an open set $\mathcal{V} \subseteq C([0,1], \mathbb{R})$ with $x \notin \nu \mathcal{K}(x)$ for any $v \in(0,1)$ and all $x \in \partial \mathcal{V}$. Let $x$ be a solution of (1.1)-(1.2). Then there exists $f \in L^{1}([0,1], \mathbb{R})$ with $f \in S_{F, x}$ such that for $t \in[0,1]$, we have

$$
\begin{aligned}
x(t)= & v \int_{0}^{t} \frac{(t-s)^{q-1}}{\Gamma(q)} f(s) d s+v \frac{t^{n-1}}{\Delta}\left(a \int_{0}^{\xi} \frac{(\xi-s)^{q}}{\Gamma(q+1)} f(s) d s\right. \\
& \left.+b \sum_{i=1}^{m-2} \alpha_{i} \int_{0}^{\eta_{i}} \frac{\left(\eta_{i}-s\right)^{q-1}}{\Gamma(q)} f(s) d s-\int_{0}^{1} \frac{(1-s)^{q-1}}{\Gamma(q)} f(s) d s\right) .
\end{aligned}
$$


Using $\left(H_{2}\right)$, we obtain

$$
|x(t)| \leq \frac{\chi(\|x\|)\|k\|}{\Gamma(q+1)}\left(1+\frac{1}{|\Delta|}\left[1+|a| \frac{\xi^{q+1}}{(q+1)}+|b| \sum_{i=1}^{m-2} \alpha_{i} \eta_{i}^{q}\right]\right),
$$

which implies

$$
\frac{\|x\|}{\chi(\|x\|)\|k\|}\left[\frac{1}{\Gamma(q+1)}\left\{1+\frac{1}{|\Delta|}\left(1+|a| \frac{\xi^{q+1}}{(q+1)}+|b| \sum_{i=1}^{m-2} \alpha_{i} \eta_{i}^{q}\right)\right\}\right]^{-1} \leq 1 .
$$

By the assumption $\left(H_{3}\right)$, there exists $N>0$ such that $\|x\| \neq N$. Let us take $\mathcal{V}=$ $\{x \in C([0,1], \mathbb{R}):\|x\|<N+1\}$. Note that the operator $\mathcal{K}: \overline{\mathcal{V}} \rightarrow \mathcal{P}(C([0,1], \mathbb{R}))$ is upper semicontinuous and completely continuous. From the choice of $\mathcal{V}$, there is no $x \in \partial \mathcal{V}$ such that $x \in \mathcal{V} \mathcal{K}(x)$ for some $v \in(0,1)$. Therefore, by Leray-Schauder alternative, it follows that the operator $\mathcal{K}$ has a fixed point $x \in \overline{\mathcal{V}}$, which is a solution of the problem (1.1)-(1.2). This completes the proof.

\subsection{Nonconvex-valued (Lipschitz) case}

In this part, we discuss the existence of solutions for the inclusion problem (1.1)(1.2) with the right-hand side being nonconvex set-valued map by applying Wegrzyk's fixed point theorem.

Let $(X, d)$ be a metric space induced from the normed space $(X ;\|\cdot\|)$. Consider $Q_{d}: \mathcal{P}(X) \times \mathcal{P}(X) \rightarrow \mathbb{R} \bigcup\{\infty\}$ given by

$$
Q_{d}(A, B)=\max \left\{\sup _{a \in A} d(a, B), \sup _{b \in B} d(b, A)\right\},
$$

where $d(a, B)=\inf _{b \in B} d(a, b)$. The map $Q_{d}$ is the (generalized) Pompeiu-Hausdorff functional. Clearly, $\left(\mathcal{P}_{b, c l}(X), Q_{d}\right)$ is a metric space and $\left(P_{d}(X), Q_{d}\right)$ is a generalized metric space.

Definition 6. A function $\Omega: \mathbb{R}^{+} \rightarrow \mathbb{R}^{+}$is said to be a strict comparison function if it is continuous strictly increasing and $\sum_{n=1}^{\infty} \Omega^{n}(t)<\infty$ for all $t>0$.

Definition 7. A multi-valued operator $\mathcal{A}: X \rightarrow \mathcal{P}_{c l}(X)$ is called

(a) $\epsilon$-Lipchitz if and only if there exists $\epsilon>0$ such that $Q_{d}(\mathcal{A}(X), \mathcal{A}(Y)) \leq$ $\epsilon d(x, y)$ for each $x, y \in X$.

(b) a contraction if and only if it is $\epsilon$-Lipschitz with $\epsilon<1$;

(c) a generalized contraction if and only if there is a strict comparison function $\Omega: \mathbb{R}^{+} \rightarrow \mathbb{R}^{+}$such that $Q_{d}(\mathcal{A}(x), \mathcal{A}(y)) \leq \Omega(d(x, y))$ for each $x, y \in X$.

Lemma 4 (Wegrzyk's fixed point theorem [33]). Let $(X, d)$ be a complete metric space. If $\mathcal{A}: X \rightarrow \mathcal{P}_{c l}(X)$ is a generalized contraction, then the operator $\mathcal{A}$ has at least one fixed point. 
Theorem 2. Assume that the following conditions hold:

$\left(H_{4}\right) \quad F:[0,1] \times \mathbb{R} \rightarrow \mathcal{P}_{c p}(\mathbb{R})$ is such that $F(., x):[0,1] \rightarrow \mathcal{P}_{c p}(\mathbb{R})$ is measurable for each $x \in \mathbb{R}$.

$\left(H_{5}\right) Q_{d}(F(t, x), F(t, \bar{x})) \leq \gamma(t) \Omega(\|x-\bar{x}\|)$ for almost all $t \in[0,1]$ and $x, \bar{x} \in \mathbb{R}$ with a function $\gamma \in C\left([0,1], \mathbb{R}^{+}\right)$and $d(0, F(t, 0)) \leq \gamma(t)$ for almost all $t \in$ $[0,1]$, where $\Omega: \mathbb{R}^{+} \rightarrow \mathbb{R}^{+}$is strictly increasing.

Then the problem (1.1)-(1.2) has at least one solution on $[0,1]$ if $\sigma \Omega: \mathbb{R}^{+} \rightarrow \mathbb{R}^{+}$is a strict comparison function, where

$$
\sigma=\frac{\|\gamma\|}{\Gamma(q+1)}\left(1+\frac{1}{|\Delta|}\left[1+|a| \frac{\xi^{q+1}}{(q+1)}+|b| \sum_{i=1}^{m-2} \alpha_{i} \eta_{i}^{q}\right]\right) .
$$

Proof. Assume that $\sigma \Omega: \mathbb{R}^{+} \rightarrow \mathbb{R}^{+}$is a strict comparison function. Then, in view of $\left(H_{4}\right)$ and $\left(H_{5}\right), F(\cdot, x(\cdot))$ is measurable and has a measurable selection $\zeta(\cdot)$ (see Theorem III.6 [11]). Also, $\gamma \in C\left([0,1], \mathbb{R}^{+}\right)$and we have

$$
\begin{aligned}
|\zeta(t)| & \leq d(0, F(t, 0))+Q_{d}(F(t, 0), F(t, x(t))) \\
& \leq \gamma(t)+\gamma(t) \Omega(|x(t)|) \leq(1+\Omega(\|x\|)) \gamma(t) .
\end{aligned}
$$

Hence, the set $S_{F, x}$ is nonempty for each $x \in C([0,1], \mathbb{R})$. Now, we will show that the operator $\mathcal{K}$ which is given by (3.2), satisfies the assumptions of Wegrzyk's fixed point Theorem. First, we show that $\mathcal{K}(x) \in \mathcal{P}_{c l}(C([0,1], \mathbb{R}))$ for each $x \in C([0,1], \mathbb{R})$. So, let, $\left\{g_{n}\right\}_{n \geq 0} \in \mathcal{K}(x)$ be such that $g_{n} \rightarrow g$ as $n \rightarrow \infty$ in $C([0,1], \mathbb{R})$. Thus $g \in$ $C([0,1], \mathbb{R})$, and there exists $\zeta_{n} \in S_{F, x_{n}}$, such that, for all $t \in[0,1]$, we have

$$
\begin{aligned}
g_{n}(t)= & \int_{0}^{t} \frac{(t-s)^{q-1}}{\Gamma(q)} \zeta_{n}(s) d s+\frac{t^{n-1}}{\Delta}\left(a \int_{0}^{\xi} \frac{(\xi-s)^{q}}{\Gamma(q+1)} \zeta_{n}(s) d s\right. \\
& \left.+b \sum_{i=1}^{m-2} \alpha_{i} \int_{0}^{\eta_{i}} \frac{\left(\eta_{i}-s\right)^{q-1}}{\Gamma(q)} \zeta_{n}(s) d s-\int_{0}^{1} \frac{(1-s)^{q-1}}{\Gamma(q)} \zeta_{n}(s) d s\right) .
\end{aligned}
$$

Since $F$ has compact values, we take a subsequence to obtain that $\zeta_{n}$ converges to $\zeta$ in $L^{1}([0,1], \mathbb{R})$. Hence, $\zeta \in S_{F, x}$, and for each $t \in[0,1]$, we have,

$$
\begin{aligned}
g_{n}(t) \rightarrow g(t)= & \int_{0}^{t} \frac{(t-s)^{q-1}}{\Gamma(q)} \zeta(s) d s+\frac{t^{n-1}}{\Delta}\left(a \int_{0}^{\xi} \frac{(\xi-s)^{q}}{\Gamma(q+1)^{2}} \zeta(s) d s\right. \\
& \left.+b \sum_{i=1}^{m-2} \alpha_{i} \int_{0}^{\eta_{i}} \frac{\left(\eta_{i}-s\right)^{q-1}}{\Gamma(q)} \zeta(s) d s-\int_{0}^{1} \frac{(1-s)^{q-1}}{\Gamma(q)} \zeta(s) d s\right) .
\end{aligned}
$$

Thus, $g \in \mathcal{K}(x)$. 
Now, we show that $Q_{d}(\mathcal{K}(x), \mathcal{K}(\bar{x})) \leq \sigma \Omega(\|x-\bar{x}\|)$, for all $x, \bar{x} \in C([0,1], \mathbb{R})$. Let $x, \bar{x} \in C([0,1], \mathbb{R})$ and $z_{1} \in \mathcal{K}(x)$, then, there exists $\zeta_{1}(t) \in S_{F, x}$ such that, for all $t \in[0,1]$, we have

$$
\begin{aligned}
z_{1}(t)= & \int_{0}^{t} \frac{(t-s)^{q-1}}{\Gamma(q)} \zeta_{1}(s) d s+\frac{t^{n-1}}{\Delta}\left(a \int_{0}^{\xi} \frac{(\xi-s)^{q}}{\Gamma(q+1)} \zeta_{1}(s) d s\right. \\
& \left.+b \sum_{i=1}^{m-2} \alpha_{i} \int_{0}^{\eta_{i}} \frac{\left(\eta_{i}-s\right)^{q-1}}{\Gamma(q)} \zeta_{1}(s) d s-\int_{0}^{1} \frac{(1-s)^{q-1}}{\Gamma(q)} \zeta_{1}(s) d s\right) .
\end{aligned}
$$

In view of the assumption $\left(H_{5}\right)$, we have $Q_{d}(F(t, x), F(t, \bar{x})) \leq \gamma(t) \Omega(\mid x(t)-$ $\bar{x}(t) \mid)$. Thus, there exists $\mu \in F(t, \bar{x}(t))$ such that

$$
\left|\zeta_{1}(t)-\mu\right| \leq \gamma(t) \Omega(|x(t)-\bar{x}(t)|), t \in[0,1] .
$$

Let us define the map, $W:[0,1] \rightarrow \mathcal{P}(\mathbb{R})$ by

$$
W(t)=\left\{\mu \in \mathbb{R}:\left|\zeta_{1}(t)-\mu\right| \leq \gamma(t) \Omega(|x(t)-\bar{x}(t)|)\right\} .
$$

Because the nonempty closed set-valued operator $W(t) \bigcap F(t, \bar{x}(t))$ is measurable (Proposition III.4 [11]), there exists a function $\zeta_{2}(t)$ which is a measurable selection for $W(t) \bigcap F(t, \bar{x}(t))$. Hence, $\zeta_{2}(t) \in F(t, \bar{x}(t))$, and $\left|\zeta_{1}(t)-\zeta_{2}(t)\right| \leq \gamma(t) \Omega(\mid x(t)-$ $\bar{x}(t) \mid)$ for each $t \in[0,1]$.

Define for each $t \in[0,1]$,

$$
\begin{aligned}
z_{2}(t)= & \int_{0}^{t} \frac{(t-s)^{q-1}}{\Gamma(q)} \zeta_{2}(s) d s+\frac{t^{n-1}}{\Delta}\left(a \int_{0}^{\xi} \frac{(\xi-s)^{q}}{\Gamma(q+1)} \zeta_{2}(s) d s\right. \\
& \left.+b \sum_{i=1}^{m-2} \alpha_{i} \int_{0}^{\eta_{i}} \frac{\left(\eta_{i}-s\right)^{q-1}}{\Gamma(q)} \zeta_{2}(s) d s-\int_{0}^{1} \frac{(1-s)^{q-1}}{\Gamma(q)} \zeta_{2}(s) d s\right) .
\end{aligned}
$$

So, by the assumption $\left(H_{5}\right)$, we obtain

$$
\begin{aligned}
& \left|z_{1}(t)-z_{2}(t)\right| \\
& \leq \int_{0}^{t} \frac{(t-s)^{q-1}}{\Gamma(q)}\left|\zeta_{1}(t)-\zeta_{2}(t)\right| d s+\frac{t^{n-1}}{|\Delta|}\left(|a| \int_{0}^{\xi} \frac{(\xi-s)^{q}}{\Gamma(q+1)}\left|\zeta_{1}(t)-\zeta_{2}(t)\right| d s\right. \\
& \quad+|b| \sum_{i=1}^{m-2} \alpha_{i} \int_{0}^{\eta_{i}} \frac{\left(\eta_{i}-s\right)^{q-1}}{\Gamma(q)}\left|\zeta_{1}(t)-\zeta_{2}(t)\right| d s \\
& \left.\quad+\int_{0}^{1} \frac{(1-s)^{q-1}}{\Gamma(q)}\left|\zeta_{1}(t)-\zeta_{2}(t)\right| d s\right),
\end{aligned}
$$


which implies that

$$
\left\|z_{1}-z_{2}\right\| \leq \frac{\|\gamma\| \Omega(\|x-\bar{x}\|)}{\Gamma(q+1)}\left(1+\frac{1}{|\Delta|}\left[1+|a| \frac{\xi^{q+1}}{(q+1)}+|b| \sum_{i=1}^{m-2} \alpha_{i} \eta_{i}^{q}\right]\right) .
$$

Interchanging the roles of $x$ and $\bar{x}$, we obtain

$$
\begin{aligned}
& Q_{d}(\mathcal{K}(x), \mathcal{K}(\bar{x})) \leq \sigma \Omega(\|x-\bar{x}\|) \\
& =\frac{\|\gamma\| \Omega(\|x-\bar{x}\|)}{\Gamma(q+1)}\left(1+\frac{1}{|\Delta|}\left[1+|a| \frac{\xi^{q+1}}{(q+1)}+|b| \sum_{i=1}^{m-2} \alpha_{i} \eta_{i}^{q}\right]\right),
\end{aligned}
$$

for all $x, \bar{x} \in C([0,1], \mathbb{R})$. Thus, the operator $\mathcal{K}$ is a generalized contraction. Therefore, by Wegrzyk's fixed point Theorem, the operator $\mathcal{K}$ has at least one fixed point $x$, which is a solution of the problem (1.1)-(1.2). This completes the proof.

Remark 2. We emphasize that the existence result (Theorem 2) obtained by applying Wegrzyk's theorem holds for several choices of the strictly increasing function $\Omega$ involved in its hypothesis. For instance, by taking $\Omega(x)=x$, we obtain a special case which is usually obtained by applying a fixed point theorem due to Covitz and Nadler [12].

\subsection{Examples}

Example 1. (Convex-valued case) Consider the following multi-valued fractional boundary value problem:

$$
\left\{\begin{array}{l}
{ }^{c} D^{5 / 4} \in F(t, x(t)), \quad t \in[0,1] \\
x(0)=0, x(1)=\int_{0}^{1 / 8} x(s) d s+\sum_{i=1}^{4} \alpha_{i} x\left(\eta_{i}\right)
\end{array}\right.
$$

where $q=5 / 4, a=b=1, \xi=1 / 8, \eta_{1}=1 / 6, \eta_{2}=1 / 3, \eta_{3}=1 / 2, \eta_{4}=2 / 3, \alpha_{1}=$ $1 / 7, \alpha_{2}=2 / 7, \alpha_{3}=3 / 7, \alpha_{4}=4 / 7$ and

$$
F(t, x(t))=\left[\frac{|x|}{9(|x|+5)}+\frac{1}{18} \cos ^{2} t, \frac{1}{2} e^{-t} \sin x\right] .
$$

Using the given data, we get

$$
\begin{gathered}
|\Delta|=\left|1-\frac{a \xi^{2}}{2}-b \sum_{i=1}^{4} \alpha_{i} \eta_{i}\right|=0.2779 \\
\frac{1}{\Gamma(q+1)}\left\{1+\frac{1}{|\Delta|}\left(1+|a| \frac{\xi^{q+1}}{q+1}+|b| \sum_{i=1}^{m-2} \alpha_{i} \eta_{i}^{q}\right)\right\}=6.0155 .
\end{gathered}
$$


Moreover, for $f \in F$, we have

$$
|f| \leq \max \left\{\frac{|x|}{9(|x|+5)}+\frac{1}{18} \cos ^{2} t, \frac{1}{2} e^{-t} \sin x\right\}, x \in \mathbb{R}, t \in[0,1] .
$$

Thus

$$
\|F(t, x)\|_{\mathcal{P}}:=\sup \{|y|: y \in F(t, x)\} \leq \frac{1}{2}, x \in \mathbb{R},
$$

with $k(t)=1, \chi(\|x\|)=1 / 2$. Next, using the condition $\left(H_{3}\right)$, we find that $N>$ $N_{1} \simeq 3.0078$. Thus, all the conditions of Theorem 1 are satisfied and consequently, the problem (3.4) with $F(t, x)$ given by (3.5), has at least one solution on $[0,1]$.

Example 2. (Nonconvex-valued case) Consider the problem (3.4) that is given in Example 1, with

$$
F(t, x(t))=\left[\frac{1}{\sqrt{625+t^{2}}}, \frac{\sin x+\frac{2}{\pi} \tan ^{-1} x}{2(4+t(1-t))^{2}}+\frac{4}{81}\right] .
$$

So, we find that

$$
\begin{gathered}
\sup \{|g|: g \in F(t, x)\} \leq \frac{1}{(4+t(1-t))^{2}}+\frac{4}{81}, \\
Q_{d}(F(t, x), F(t, \bar{x})) \leq \frac{1}{(4+t(1-t))^{2}}\left[\frac{(2+\pi)}{2 \pi}\|x-\bar{x}\|\right] .
\end{gathered}
$$

Put $\gamma(t)=\frac{1}{(4+t(1-t))^{2}}$, so, $\|\gamma\|=1 / 16$. Thus, we get

$$
\sigma=6.0155\|\gamma\|=6.0155 / 16 \simeq 0.37597,
$$

and $Q_{d}(F(t, x), F(t, \bar{x})) \leq \gamma(t) \Omega(\|x-\bar{x}\|)$, where $\Omega(\|x-\bar{x}\|)=\frac{(2+\pi)}{2 \pi}\|x-\bar{x}\|$. Hence, all the conditions of Theorem 2 are satisfied. Therefore, the problem (3.4) with $F(t, x)$ given by (3.6), has at least one solution on $[0,1]$.

\section{ACKNOWLEDGEMENT}

We appreciate the reviewer for his/her constructive remarks that led to the improvement of the original manuscript.

\section{REFERENCES}

[1] S. Adly, T. Haddad, and L. Thibault, "Convex sweeping process in the framework of measure differential inclusions and evolution variational inequalities," Math. Program, Ser. B, vol. 148, pp. 5-47, 2014.

[2] B. Ahmad, "Sharp estimates for the unique solution of two-point fractional-order boundary value problems," Appl. Math.Lett., vol. 65, pp. 77-82, 2017, doi: 10.1016/j.aml.2016.10.008.

[3] B. Ahmad, T. Hayat, and S. Asghar, "Diffraction of a plane wave by an elastic knife-edge adjacent to a strip," Canad. App. Math. Quart., vol. 9, pp. 303-316, 2001. 
[4] B. Ahmad and S. Ntouyas, "Existence results for a coupled system of Caputo type sequential fractional differential equations with nonlocal integral boundary conditions," Appl. Math. Comput., vol. 266, pp. 615-622, 2015, doi: 10.1016/j.amc.2015.05.116.

[5] B. Ahmad and S. Ntouyas, "Nonlocal fractional boundary value problems with slit-strips boundary conditions," Fract. Calc. Appl. Anal., vol. 18, pp. 261-280, 2015.

[6] B. Ahmad and S. Ntouyas, "On perturbed fractional differential inclusions with nonlocal multipoint Erdelyi-Kober fractional integral boundary conditions," Mediterr. J. Math., vol. 14:27, 2017, doi: 10.1007/s00009-016-0830-5.

[7] A. Alsaedi, S. Ntouyas, B. Ahmad, and H. Alsulami, "Existence results for fractional integral inclusions via nonlinear alternative for contractive maps," Bound. Value Probl., vol. 25, p. 14p., 2014, doi: 10.1186/1687-2770-2014-25.

[8] S. Asghar, B. Ahmad, and M. Ayub, "Diffraction from an absorbing half plane due to a finite cylindrical source," Acustica-Acta Acustica, vol. 82, pp. 365-367, 1996.

[9] S. Balochian and M. Nazari, "Stability of particular class of fractional differential inclusion systems with input delay," Control Intell. Syst., vol. 42, pp. 279-283, 2014.

[10] J. Bastien, "Study of a driven and braked wheel using maximal monotone differential inclusions: applications to the nonlinear dynamics of wheeled vehicles," Archive of Applied Mechanics, vol. 84, pp. 851-880, 2014, doi: 10.1007/s00419-014-0837-y.

[11] C. Castaing and M. Valadier, Convex Analysis and Measurable Multifunctions. BerlinHeidelberg-New York: Springer-Verlag, 1997.

[12] H. Covitz and S. Nadler, "Multivalued contraction mappings in generalized metric spaces," Isr. J. Math., vol. 8, pp. 5-11, 1970.

[13] M.-F. Danca, "Synchronization of piecewise continuous systems of fractional order," Nonlinear Dynam., vol. 78, pp. 2065-2084, 2014, doi: 10.1007/s11071-014-1577-9.

[14] K. Deimling, Multivalued Differential Equations. Berlin-New York: Walter De Gruyter, 1992.

[15] Y. Ding, Z. Wei, J. Xu, and D. O'Regan, "Extremal solutions for nonlinear fractional boundary value problems with $p$-Laplacian," J. Comput. Appl. Math., vol. 288, pp. 151-158, 2015, doi: 10.1016/j.cam.2015.04.002.

[16] J. Edmond and L. Thibault, "Relaxation of an optimal control problem involving a perturbed sweeping process," Math. Program, Ser. B, vol. 104, pp. 347-373, 2005.

[17] A. Granas and J. Dugundji, Fixed Point Theory. New York: Springer-Verlag, 2005.

[18] J. Henderson and N. Kosmatov, "Eigenvalue comparison for fractional boundary value problems with the Caputo derivative," Fract. Calc. Appl. Anal., vol. 17, pp. 872-880, 2014, doi: 10.2478/s13540-014-0202-4.

[19] S. Hu and N. Papageorgiou, Handbook of Multivalued Analysis, Volume I: Theory. Kluwer: Dordrecht, 1997.

[20] A. Kilbas, H. Srivastava, and J. Trujillo, Theory and Applications of Fractional Differential Equations. Amsterdam: Elsevier Science, 2006.

[21] M. Korda, D. Henrion, and C. Jones, "Convex computation of the maximum controlled invariant set for polynomial control systems," SIAM J. Control Optim., vol. 52, pp. 2944-2969, 2014, doi: $10.1137 / 130914565$.

[22] A. Lasota and Z. Opial, "An application of the Kakutani-Ky Fan theorem in the theory of ordinary differential equations,” Bull. Acad. Polon. Sci. Ser.Sci. Math. Astronom. Phys., vol. 13, pp. 781$786,1965$.

[23] X. Liu, Z. Liu, and X. Fu, "Relaxation in nonconvex optimal control problems described by fractional differential equations," J. Math. Anal. Appl., vol. 409, pp. 446-458, 2014, doi: 10.1186/1687-2770-2014-25.

[24] M. Kisielewicz, Stochastic Differential Inclusions and Applications. Springer Optimization and Its Applications. New York: Springer, 2013. 
[25] N. I. Mahmudov, R. Murugesu, C. Ravichandran, and V. Vijayakumar, "Approximate controllability results for fractional semilinear integro-differential inclusions in Hilbert spaces," Results Math., vol. 71, pp. 45-61, 2017, doi: 10.1007/s00025-016-0621-0.

[26] M. Monteiro and D. Manuel, Differential Inclusions in Nonsmooth Mechanical Problems. Shocks and Dry Friction. Progress in Nonlinear Differential Equations and their Applications. Basel: Birkhauser Verlag, 1993.

[27] S. Ntouyas, Nonlocal initial and boundary value problems a survey. Handbook on Differential Equations: Ordinary Differential Equations, Edited by A. Canada, P. Drabek and A. Fonda,. New York: Elsevier Science, 2005.

[28] S. Ntouyas, S. Etemad, and J. Tariboon, "Existence results for multi-term fractional differential inclusions," Adv. Difference Equ., vol. 2015:140, 2015, doi: 10.1186/s13662-015-0481-z.

[29] D. Qarout, B. Ahmad, and A. Alsaedi, "Existence theorems for semi-linear Caputo fractional differential equations with nonlocal discrete and integral boundary conditions," Fract. Calc. Appl. Anal., vol. 19, pp. 463-479, 2015, doi: 10.1515/fca-2016-0024.

[30] P. J. Quezada, L. Sagnol, and C. Chazallon, "Shear test on viscoelastic granular material using Contact Dynamics simulations," EPJ Web of Conferences, vol. 140, 2017, doi: https://doi.org/10.1051/epjconf/201714008009.

[31] V. Recupero, "A continuity method for sweeping processes," J. Differential Equations, vol. 251, pp. 2125-2142, 2011, doi: 10.1016/j.jde.2011.06.018.

[32] P. Richard, M. Nicodemi, R. Delannay, P. Ribiere, and D. Bideau, "Slow relaxation and compaction of granular system," Nature Mater, vol. 4, pp. 121-128, 2005, doi: 10.1088/0953$8984 / 17 / 24 / 024$.

[33] R. Wegrzyk, "Fixed point theorems for multifunctions and their applications to functional equations," Dissertationes Math. (Rozprawy Mat.), vol. 201, 1982.

[34] Z.-B. Wu, Y.-Z. Zou, and N.-J. Huang, "A class of global fractional-order projective dynamical systems involving set-valued perturbations," Appl. Math. Comput., vol. 277, pp. 23-33, 2016, doi: 10.1016/j.amc.2015.12.033.

[35] Y. Zhou, Basic Theory of Fractional Differential Equations. New Jersey: World Scientific Publishing Co. Pte. Ltd, 2014.

\section{Authors' addresses}

\section{Bashir Ahmad}

Nonlinear Analysis and Applied Mathematics (NAAM)-Research Group, Department of Mathematics, Faculty of Science, King Abdulaziz University, P.O. Box 80203, Jeddah 21589, Saudi Arabia

E-mail address: bashirahmad-qaulyahoo.com

\section{Doa'a Garout}

Nonlinear Analysis and Applied Mathematics (NAAM)-Research Group, Department of Mathematics, Faculty of Science, King Abdulaziz University, P.O. Box 80203, Jeddah 21589, Saudi Arabia

E-mail address: dgarout@kau.edu.sa

\section{Sotiris K. Ntouyas}

University of Ioannina, Department of Mathematics, 45110 Ioannina, Greece and, Nonlinear Analysis and Applied Mathematics (NAAM)-Research Group, Department of Mathematics, Faculty of Science, King Abdulaziz University, P.O. Box 80203, Jeddah 21589, Saudi Arabia

E-mail address: sntouyas@uoi.gr 
Ahmed Alsaedi

Nonlinear Analysis and Applied Mathematics (NAAM)-Research Group, Department of Mathematics, Faculty of Science, King Abdulaziz University, P.O. Box 80203, Jeddah 21589, Saudi Arabia

E-mail address: aalsaedi@hotmail.com 